\title{
AN ISOMORPHISM FOR THE GROTHENDIECK RING OF A HOPF ALGEBRA ORDER
}

\author{
ANNA-LISE JENSEN AND RICHARD G. LARSON
}

\begin{abstract}
If $G$ is a finite abelian group, $R$ is a principal ideal domain with field of quotients an algebraic number field $K$ which splits $G$, and if $A$ is a Hopf algebra order in $K G$, then the Grothendieck ring of the category of finitely generated $A$-modules is isomorphic to the Grothendieck ring of the category of finitely generated $R G$-modules.
\end{abstract}

The Grothendieck group ${ }_{A} \mathcal{G}$ of the category of finitely generated modules over a ring $A$ is analogous to the free group generated by the characters of a finite group. If $H$ is a Hopf algebra, then ${ }_{H} \mathcal{G}$ is a ring. We show if $R$ is a principal ideal domain with field of quotients $K$ which splits $G$, if $G$ is a finite abelian group, and if $A$ is a Hopf algebra order in $K G$, then ${ }_{R G} \mathcal{G} \simeq{ }_{A} \mathcal{G}$. Swan [6] has shown that if $G$ is a finite group, and if $R$ is a semilocal Dedekind domain, then ${ }_{R G} \mathcal{G} \simeq{ }_{K G} \mathcal{G}$. It follows that if $R$ is a semilocal Dedekind domain with field of quotients $K$, if $G$ is a finite abelian group split by $K$, and if $A$ is a Hopf algebra order in $K G$, then ${ }_{A} \mathcal{G} \simeq{ }_{K G} \mathcal{G}$. Swan's result says that, factoring out relations induced by short exact sequences of representations, the representation theory of a group ring over a semilocal Dedekind domain is the same as the representation theory of the group algebra over the field of quotients. Our result says that, for abelian groups, the representation theory is the same for any Hopf algebra order in the group algebra as for the group ring.

If $C$ is an abelian category, the Grothendieck group of $C$ is defined as follows: for each object $M \in C$ there is a generator $[M]$; for each short exact sequence

$$
0 \rightarrow M^{\prime} \rightarrow M \rightarrow M^{\prime \prime} \rightarrow 0
$$

in $C$ there is a relation

$$
[M]=\left[M^{\prime}\right]+\left[M^{\prime \prime}\right] .
$$

For the remainder of this paper $R$ will be a principal ideal domain with field of quotients an algebraic number field $K$. If $A$ is an $R$-algebra which is a finitely generated projective $R$-module, we will denote by ${ }_{A} C$ the category of finitely generated $A$-modules, and by ${ }_{A} C$ the category of finitely generated $A$-modules which are torsion free as $R$-modules. We will denote the Grothendieck group of ${ }_{A} C$ by ${ }_{A} \mathcal{G}$, and the Grothendieck group of ${ }_{A} \mathrm{C}$ by ${ }_{A} \mathcal{G}$. The embedding ${ }_{A} \mathrm{C} \rightarrow{ }_{A} \mathrm{C}$ induces an isomorphism ${ }_{A} \mathcal{G} \rightarrow{ }_{A} \mathcal{G}$. (See [4] for details.)

If $H$ is a Hopf algebra over $R$, and $M, N \in{ }_{H} C$, then $M \otimes_{R} N \in{ }_{H \otimes_{R} H} C$. Pullback along the coproduct $\delta: H \rightarrow H \otimes_{R} H$ gives an $H$-module structure on $M \otimes_{R} N$. In this manner $\otimes_{R}$ gives rise to a ring structure on ${ }_{H} \mathcal{G}$ with multiplication given by

$$
[M][N]=\left[M \otimes_{R} N\right] .
$$

Received by the editors May 21, 1984.

1980 Mathematics Subject Classification. Primary 16A24, 16A54.

Key words and phrases. Grothendieck group, Hopf algebra order, representation. 
THEOREM 1. Let $G$ be a finite abelian group. Let $R$ be a principal ideal domain with field of quotients an algebraic number field $K$ which splits $G$. Let $A$ be a Hopf algebra order in $K G$. If $M$ is an A-module which is a finitely generated torsion $R$-module, then $[M]=0$ in ${ }_{A} \mathcal{G}$.

PROOF. If $p$ is a prime in $R$, let

$$
M^{p}=\left\{x \in M \mid p^{r} x=0 \text { for some } r>0\right\} .
$$

Then

$$
M=\bigoplus_{p} M^{p},
$$

where the sum ranges over all primes $p$ in $R$. We see that $[M]=0$ if each $\left[M^{p}\right]=0$. Therefore, since $M$ is a finitely generated $R$-module, we may assume that $p^{r} M=0$ for some prime $p \in R$. Looking at the short exact sequence

$$
0 \rightarrow p M \rightarrow M \rightarrow M / p M \rightarrow 0
$$

and using induction on $r$ and the fact that $p^{r-1}(p M)=0$, we see that it is sufficient to show that $p M=0$ implies $[M]=0$. In other words, we may assume that $M$ is a vector space over the field $k=R / p R$. Since $M$ is a finitely generated $R$-module, $\operatorname{dim}_{k} M$ is finite. If $N$ is a proper $A$-submodule of $M$, then both $N$ and $M / N$ are $A$-modules which are vector spaces over $k$ of smaller dimension than $M$, and $[M]=[N]+[M / N]$. Therefore, we may assume that $M$ is an irreducible $A$-module.

We first show that $\operatorname{dim}_{k} M=1$. Let $R_{p}$ be the localization of $R$ at the prime $(p)$, and let $A_{p}=R_{p} \otimes_{R} A$. We have that $A_{p}$ is a Hopf algebra order over $R_{p}$ in $K G$, and that $M$ is an irreducible module over $A_{p}$. Let $A_{p}^{*}=\operatorname{hom}_{R_{p}}\left(A_{p}, R_{p}\right)$. Since $K$ splits $G, A_{p}^{*}$ is a Hopf algebra order in $K \hat{G}$. If $X$ is a $R$-module (respectively a $R_{p}$-module) denote by $\bar{X}$ the $k$-space $k \otimes_{R} X$ (respectively $k \otimes_{R_{p}} X$ ). Note that if $X$ is an $R$-module, $k \otimes_{R} X \simeq k \otimes_{R_{p}}\left(R_{p} \otimes_{R} X\right)$. Following the notation and results of $[3, \S 3]$, we have a sequence of Hopf algebra maps

$$
A_{p+}^{*} \rightarrow A_{p}^{*} \rightarrow R_{p} \hat{G} / \hat{G}_{+},
$$

which induces a decomposition

$$
\bar{A}^{*} \simeq \bar{A}_{p}^{*} \simeq \bar{A}_{p+}^{*} \# k \hat{G} / \hat{G}_{+} .
$$

Let $S=\left(R_{p} \hat{G} / \hat{G}_{+}\right)^{*}$. Then $S \subset A_{p}$ is a separable Hopf algebra over $R_{p}$. Since $K$ splits $G$ and $\hat{G}$,

$$
S=\bigoplus_{i} e_{i} R_{p}
$$

for orthogonal idempotents $e_{i}$. We also have that $A_{p}=\bigoplus_{i} e_{i} A_{p}$ as rings. Each $e_{i} M$ is an $A_{p}$-module, and an $e_{i} A_{p}$-module. The facts that $M$ is an irreducible $A_{p^{-}}$ module and that $M=\bigoplus e_{i} M$ imply that $M=e_{i} M$ for some $i$. This implies that $M$ is an irreducible $e_{i} A_{p}$-module. Suppose that the idempotents $e_{i}$ are numbered so that $\epsilon\left(e_{0}\right)=1$, and $\epsilon\left(e_{i}\right)=0$ for $i \neq 0$. Then $e_{0} A_{p} \simeq\left(A_{p+}^{*}\right)^{*}$. We have an action of $A_{p}^{*}$ on $A_{p}$ defined by $f \cdot a=\sum f\left(a_{(2)}\right) a_{(1)}$, where $f \in A_{p}^{*}, a \in A_{p}$, and $\delta(a)=\sum a_{(1)} \otimes a_{(2)}$. If $\delta(f)=\sum f_{(1)} \otimes f_{(2)}$, then $f \cdot(a b)=\sum\left(f_{(1)} \cdot a\right)\left(f_{(2)} \cdot b\right)$. In particular, if $f \in \Gamma\left(A_{p}^{*}\right)$, the map $a \mapsto f \cdot a$ is an algebra isomorphism. since $\Gamma\left(S^{*}\right)=\hat{G} / \hat{G}_{+}$is a quotient group of $\Gamma\left(A_{p}^{*}\right)=\hat{G}$, we can find $\chi_{i} \in \Gamma\left(A_{p}^{*}\right)$ satisfying 
$\chi_{i}\left(e_{i}\right)=1$, and $\chi_{i}\left(e_{j}\right)=0$ if $j \neq i$. Then $\chi_{i} \cdot e_{i}=e_{0}$, so $\chi_{i} \cdot\left(e_{i} A_{p}\right)=e_{0} A_{p} \simeq\left(A_{p+}^{*}\right)^{*}$. Denote the Hopf algebra $\left(A_{p+}^{*}\right)^{*}$ by $B$. The map $a \mapsto \chi_{i} \cdot\left(e_{i} a\right)$ gives an algebra homomorphism from $A_{p}$ to $B$, whose kernel annihilates $M$. Therefore we may think of $M$ as a $B$-module. In particular, $M$ is an irreducible $B$-module which satisfies $p M=0$. We have by $[3, \S 3]$ that $\Gamma\left(\bar{B}^{*}\right)=\{\epsilon\}$, so that $\bar{B}^{*}$ is a pointed cocommutative Hopf algebra, so that $\overline{B^{+}}$is nilpotent (where $B^{+}=\operatorname{ker} \epsilon$ denotes the augmentation ideal of $B$ ), so that $\left(B^{+}\right)^{t} \subset p B^{+}$for some $t>0$, so that $\left(B^{+}\right)^{t} M=0$. Since $M$ is an irreducible $B$-module, it follows that $B^{+} M=0$, so that $M$ is a trivial $B$-module. Since $M$ is an irreducible $B$-module, $\operatorname{dim}_{k} M=1$.

We now show that $[M]=0$ in ${ }_{A} \mathcal{G}$. Let $M=k m$. Then there exists an algebra homomorphism $\chi: A \rightarrow k$ with $a m=\chi(a) m$ for all $a \in A$. Note that $\chi \in \Gamma\left(\bar{A}^{*}\right)=$ $\Gamma\left(\bar{A}_{p}^{*}\right)=\hat{G} / \hat{G}_{+}$. Suppose $\chi=\phi \hat{G}_{+}$, for some $\phi \in \hat{G}$. We see that $\phi$ maps to $\chi$ under the map $\hat{G} \rightarrow \bar{A}^{*}$. Since $K$ splits $G$ and $\hat{G}$, by [2] we have that $\phi \in A^{*}$. Define $N$ to be a one dimensional free $R$-module $N=R n$ with $A$-module structure given by $a n=\phi(a) n$ for all $a \in A$. Then $N \in \underline{A} C$, and

$$
0 \rightarrow N \stackrel{\pi}{\rightarrow} N \rightarrow M \rightarrow 0,
$$

where $\pi(x)=p x$, is a short exact sequence of $A$-modules. Therefore $[M]=[N]-$ $[N]=0$ in ${ }_{A} \mathcal{G}$. This completes the proof of Theorem 1 .

THEOREM 2. Let $G$ be a finite abelian group. Let $R$ be a principal ideal domain with field of quotients an algebraic number field $K$ which splits $G$. If $A$ is a Hopf algebra order in $K G$, then ${ }_{R G} \mathcal{G} \simeq{ }_{A} \mathcal{G}$.

PROOF. If $H$ is a Hopf algebra over $R$ which is finitely generated as an $R$ module, then ${ }_{H} \mathcal{G} \simeq{ }_{H} \mathcal{G}[4]$. Therefore it is sufficient to show that ${ }_{R G} \mathcal{G} \simeq \underline{A} \mathcal{G}$. Since $K \otimes_{R} R G \simeq K \otimes_{R} A \simeq K G$ we may identify $R G$ and $A$ with their images in $K G$. If $M \in{ }_{R G} C$ then we may identify $M$ with its image in $K \otimes_{R} M$. In this case, $K \otimes_{R} \bar{M}$ is a $K G$-module in a natural fashion. Since $A \subset K G$ we can form the product $A \cdot M$, using the above identifications. We have $A \cdot M \in{ }_{A} C$. If $M, N \in{ }_{R G} C$ and $f: M \rightarrow N$ is a $R G$-module morphism, then $f$ induces an $A$-module homomorphism $\bar{f}: A \cdot M \rightarrow A \cdot N$. The map $\phi$ defined by $\phi: M \mapsto A \cdot M$ is a functor from ${ }_{R G} C$ to ${ }_{\underline{A}} C$.

We shall show that $\phi$ induces a ring homomorphism $\Phi:{ }_{R G} \mathcal{G} \rightarrow \underline{A} \mathcal{G}$ and that $\Phi$ is an isomorphism. We first show that $\phi$ preserves the defining relations for $\underline{R G} \mathcal{G}$. If $[M]=\left[M^{\prime}\right]+\left[M^{\prime \prime}\right]$ is the relation in $\underline{R G} \mathcal{G}$ corresponding to the short exact sequence

$$
0 \rightarrow M^{\prime} \stackrel{g}{\rightarrow} M \stackrel{f}{\rightarrow} M^{\prime \prime} \rightarrow 0
$$

consider the sequence

$$
A \cdot M^{\prime} \stackrel{\bar{g}}{\rightarrow} A \cdot M \stackrel{\bar{f}}{\rightarrow} A \cdot M^{\prime \prime} .
$$

We see that $\bar{f}$ is onto. If $N=\operatorname{ker} \bar{f}$ then $\bar{g}\left(A \cdot M^{\prime}\right) \subset N$, and the short exact sequences

$$
0 \rightarrow N \rightarrow A \cdot M \rightarrow A \cdot M^{\prime \prime} \rightarrow 0
$$

and

$$
0 \rightarrow A \cdot M^{\prime} \rightarrow N \rightarrow N /\left(A \cdot M^{\prime}\right) \rightarrow 0
$$


define relations in ${ }_{A} \mathcal{G}$. Since $N / A \cdot M^{\prime}$ is a torsion $R$-module, by Theorem 1 we have that $\left[N / A \cdot M^{\prime}\right]=0$. Therefore $[N]=\left[A \cdot M^{\prime}\right]$ and $[A \cdot M]=[N]+\left[A \cdot M^{\prime \prime}\right]=$ $\left[A \cdot M^{\prime}\right]+\left[A \cdot M^{\prime \prime}\right]$. This shows that $\phi$ preserves relations. $\Phi$ is a ring homomorphism, since

$$
(A \cdot M) \otimes_{R}(A \cdot N) /\left(A \cdot\left(M \otimes_{R} N\right)\right)
$$

is a torsion $R$-module, so Theorem 1 implies

$$
\left[(A \cdot M) \otimes_{R}(A \cdot N) /\left(A \cdot\left(M \otimes_{R} N\right)\right)\right]=0
$$

in ${ }_{A} \mathcal{G}$, so

$$
[A \cdot M][A \cdot N]=\left[(A \cdot M) \otimes_{R}(A \cdot N)\right]=\left[A \cdot\left(M \otimes_{R} N\right)\right] .
$$

We now show that $\Phi$ is an isomorphism, by giving its inverse. The inverse to $\Phi$ is the map P: ${ }_{A} \mathcal{G} \rightarrow \underline{R G} \mathcal{G}$ which is induced by restriction of scalars. Let $M \in{ }_{R G} C$ and view $A \cdot M$ as an $R G$-module by restriction. The $R G$-module $A \cdot M / M$ is a torsion $R$-module, so by Theorem $1,[A \cdot M / M]=0$ in ${ }_{R G} \mathcal{G}$, so $[A \cdot M]=[M]$. This shows that $\mathrm{P} \circ \Phi$ is the identity on $R G \mathcal{G}$. A similar argument shows that $\Phi \circ \mathrm{P}$ is the identity on $\underline{A} \mathcal{G}$. Therefore $\underline{A}_{\mathcal{G}} \simeq_{\underline{R} G} \mathcal{G}$.

COROLlARY. let $G$ be a finite abelian group. let $R$ be a semilocal Dedekind domain with field of quotients an algebraic number field $K$ which splits $G$. If $A$ is a Hopf algebra order in $K G$, then ${ }_{A} \mathcal{G}$ is isomorphic to the ring of characters of $G$.

Proof. A semilocal Dedekind domain is a principal ideal domain [1]. The corollary now follows from Theorem 2 and the facts that ${ }_{R G} \mathcal{G} \simeq{ }_{K G} \mathcal{G}[6]$ and that ${ }_{K G} \mathcal{G}$ is isomorphic to the character ring of $G$.

\section{REFERENCES}

1. T. W. Hungerford, Algebra, Springer-Verlag, New York, 1974.

2. R. G. Larson, Group rings over Dedekind domains, J. Algebra 5 (1967), 358-361.

3. __ Hopf algebra orders determined by group valuations, J. Algebra 38 (1976), 414-452.

4. J. P. Serre, Groupes de Grothendieck des schémas en groupes réductifs déployés, Inst. Hautes Études Sci. Publ. Math. 34 (1968), 37-52.

5. R. G. Swan, Induced representations and projective modules, Ann. of Math. 71 (1960), 552-578.

6. __ The Grothendieck ring of a finite group, Topology 2 (1963), 85-110.

Department of Mathematics, Mundelein College, Chicago, illinois 60660

Department of Mathematics, University of Illinois, Chicago, Illinois 60680 (Current address of R. G. Larson)

Current address (Anna-Lise Jensen): Department of Mathematics, Northeastern Illinois University, Chicago, Illinois 60625 